\title{
Anterior Cruciate Ligament Sizing Tools Can Be Interchanged Without Affecting Graft Diameter Measurement
}

\author{
Maude Joannette Bourguignon, M.D., F.R.C.S.C., Sonia Bédard, \\ Frédéric Balg, M.D., F.R.C.S.C., Karina Lebel, Ing. Ph.D., \\ Alexandre Keith Leang, M.D., F.R.C.S.C., and François Vézina, M.D., F.R.C.S.C.
}

\begin{abstract}
Purpose: To determine whether different types of measurement tools can be interchanged without significantly affecting the resulting graft diameter. Methods: Hamstrings (gracilis and semitendinosus) and quadriceps tendons in 33 cadaver knees were harvested. Three different anterior cruciate ligament (ACL) graft combinations were created using these tendons, making 99 cadaver grafts samples available to measure. The grafts were randomly passed through sizing tubes and a slotted measurement block to determine their diameter. Interobserver and intraobserver reliabilities of measurements were assessed. Pearson correlation test, as well as Bland Altman graph, were used to evaluate the interchangeability of the tools. Results: In 95\% of cases, the diameter difference between the tubes and the block measures was less than the $0.5 \mathrm{~mm}$ in increment cutoff. Both the intraobserver and interobserver reliability were excellent. Conclusions: This study showed that the ACL graft diameter measurement does not vary whether a slotted block or sizing tube from the same company is used. Clinical Relevance: ACL graft size has an influence on the surgical technique and clinical outcomes. Therefore it is important to have reliable sizing tools.
\end{abstract}

A nterior cruciate ligament (ACL) reconstruction is among the most common elective procedures in Orthopaedics. In the United States alone, approximately 130,000 primary ACL reconstructions are performed each year. ${ }^{1,2}$ Autologous hamstring tendon (HT) grafts are often selected for their biomechanical properties and their size. The harvesting technique is simple and reproducible. Moreover, HT grafts can easily be folded or augmented to increase graft diameter. ${ }^{3,4}$

From the Orthopaedic Surgery, Surgery Department, Faculté de Médecine et des Sciences de la Santé, Université de Sherbrooke (M.J.B., F.B., A.K.L., F.V.), Québec; Programme de Recherche en Science de la Santé, Faculté de Médecine et des Sciences de la Santé, Université de Sherbrooke (S.B.), Québec; Centre de Recherche du CHUS, Centre Intégré Universitaire de Santé et de Services Sociaux de l'Estrie Centre Hospitalier Universitaire de Sherbrooke du Québec (S.B., F.B.), Québec; Electrical Engineering, Université de Sherbrooke (K.L.), Québec; and Centre de Recherche Sur le Vieillissement (CDRV), Centre Intégré Universitaire de Santé et de Services Sociaux de l'Estrie Centre Hospitalier Universitaire de Sherbrooke (K.L.), Québec, Canada.

The funds were granted by the surgery department of the CIUSSS de l'Estrie-CHUS and the FREOS (Fondation de Recherche et d'Enseignement en Orthopédie de Sherbrooke).

The authors report the following potential conflicts of interest or sources of funding: S.B. reports grants from DePuy; and other from Wright Medical Technology, Inc., and Zimmer. F.B. reports grants from DePuy; and other
Graft sizing is crucial for adequate bone tunnels preparation and to determine the relative risk of graft failure. It has been shown that each $0.5 \mathrm{~mm}$ increment in ACL graft diameter significantly lowers graft failure rate. $^{5,6}$ Therefore the sizing technique must be reproducible and reliable. ${ }^{7,8}$

Multiple sizing tools have been developed by several implant companies. Some ancillaries provide an open sizing block, closed tubes or both to determine the graft diameter. If an ACL reconstruction system contains

from Wright Medical Technology, Inc., and Zimmer. F.V reports grants from DePuy; and other from Wright Medical Technology, Inc., and Zimmer. Full ICMJE author disclosure forms are available for this article online, as supplementary material.

Received September 8, 2020; accepted January 3, 2022.

Address correspondence to François Vézina, M.D., F.R.C.S.C., Orthopaedic Surgery, Surgery Department, Faculté de Médecine et des Sciences de la Santé, Université de Sherbrooke, Québec; Canada. E-mail: francois.vezina@ usherbrooke.ca

(C) 2022 THE AUTHORS. Published by Elsevier Inc. on behalf of the Arthroscopy Association of North America. This is an open access article under the CC BY-NC-ND license (http://creativecommons.org/licenses/by-nc-nd/4.0/). 2666-061X/201497

https://doi.org/10.1016/j.asmr.2022.01.002 


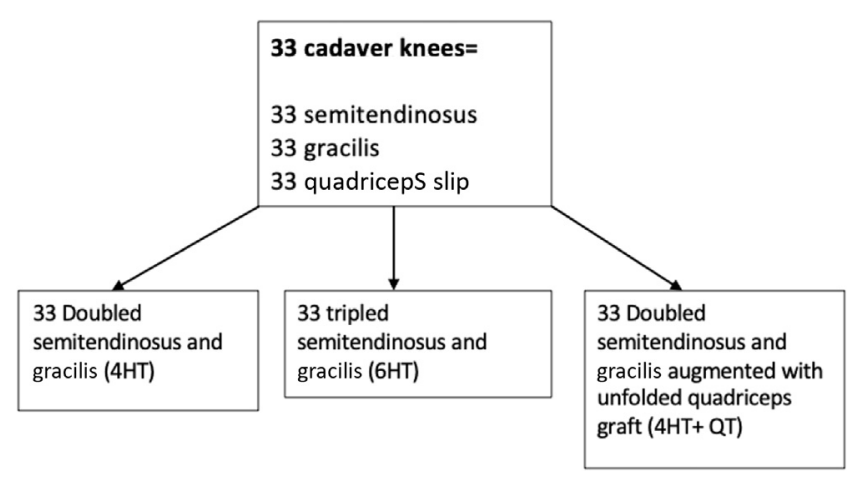

Fig 1. Graft combinations.

measurement tools of different geometry, these need to be interchangeable so that the measured graft diameter does not change according to the tool used. The purpose of this study was to determine whether different types of measurement tools can be interchanged without significantly affecting the resulting graft diameter. Our hypothesis was that given that some tendon fibers could escape through the opening, the slotted sizing block could underestimate the graft diameter.

\section{Methods}

The present study used a descriptive correlational design. The protocol was approved by the center's Ethics committee.

\section{Experimental protocol}

Between January and March 2019, tendons were harvested from 33 cadaver knees obtained through the center's cadaver laboratory. Two attending orthopaedic sports surgeons and a postgraduate year 4-level resident performed open semitendinosus, gracilis and quadricipital tendon harvests. The hamstring tendons were obtained using a standard open technique over the pes anserinus. A tendon harvester (Conmed, Largo, FL /Linvatec Corporation, Largo, FL) was used in the process. The quadriceps tendons (QT) were collected at the middle third off their insertion on the patella. All QT grafts involved only the thickness of the rectus femoris tendon and measured $6 \mathrm{~mm}$ wide $\times 9 \mathrm{~cm}$ long.

This process allowed for 3 different combinations of tendons from the same donor knee to obtain the following: (1) doubled semitendinosus and gracilis (4HT); (2) tripled semitendinosus and gracilis (6HT); (3) doubled semitendinosus and gracilis augmented with unfolded quadriceps tendon graft (4HT+QT). In total, 99 different graft samples were available for graft diameter measurement (Fig 1). In addition, 10 tendons, randomly selected by the research coordinator, were measured a second time by both surgeons for intraobserver reliability evaluation. All measurements were performed by the 2 attending surgeons who prepared the grafts (i.e., total of 109 samples measured by 2 surgeons, using 2 different measurement tools). The tendon grafts were measured using the Conmed Graft Max ACL (Conmed) (Fig 1) system, which contains a sizing block with slotted holes (open slot) and multiple sizing tubes (closed) from 6.5 to $11 \mathrm{~mm}$ in $0.5 \mathrm{~mm}$ increment.

First, the tendons were folded in halves and measured (4HT). The quadriceps tendon was then added in the middle of the folded HT as the second configuration $(4 \mathrm{HT}+\mathrm{GT})$ (Fig 2). Finally, the HT tendons were folded in thirds, braided, and measured again (6HT). To proceed with the diameter measurement, each sample was passed through the sizing block slotted holes by $0.5 \mathrm{~mm}$ decrement (Conmed Graft Max ACL system) until the smallest possible diameter was reached (Fig 3). Samples were also measured with the tubes, using the same process (Paramax ACL system; Conmed). The 2 sizing methods were attempted in no specific order. The observers were not allowed to measure the graft more than once with each sizing tool to prevent sample compression, sizing alterations, and measurement bias.

\section{Statistical Analyses}

In accordance with our first aim, intraobserver reliability was assessed using an intracorrelation coefficient (ICC). Guidelines used for interpretation assume poor reliability for ICC under 0.5 , moderate reliability between 0.51 and 0.75 , good reliability for ICC between 0.75 and 0.90 , and excellent above that threshold (Koo and Li, 2016: A Guideline of Selecting and Reporting Intraclass Correlation Coefficients for Reliability Research). Inter-rater reliability was also assessed through an ICC and interpreted following the abovementioned guidelines. Interchangeability of the two measurement tools was then assessed two ways. First, a Pearson correlation test was used to get an intuitive appreciation of the correlation. Then, the Bland-

A

Doubled semitendinosus and gracilis with unfolded quadriceps tendon ( $4 \mathrm{HT}+\mathrm{QT})$

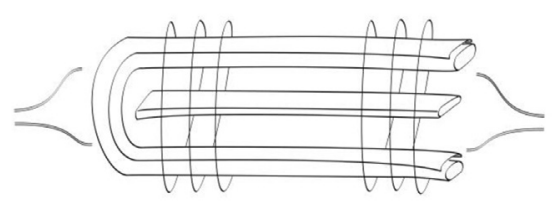

B

Tripled semitendinosus and gracilis

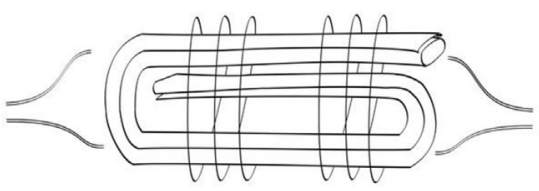

Fig 2. Graft drawing. (A) Doubled semitendinosus and gracilis with unfolded quadriceps tendon (4HT+ QT). (B) Tripled semitendinosus and gracilis. 
Fig 3. Conmed anterior cruciate ligament measurement tools: (A) Slotted block and (B) sizing tubes.

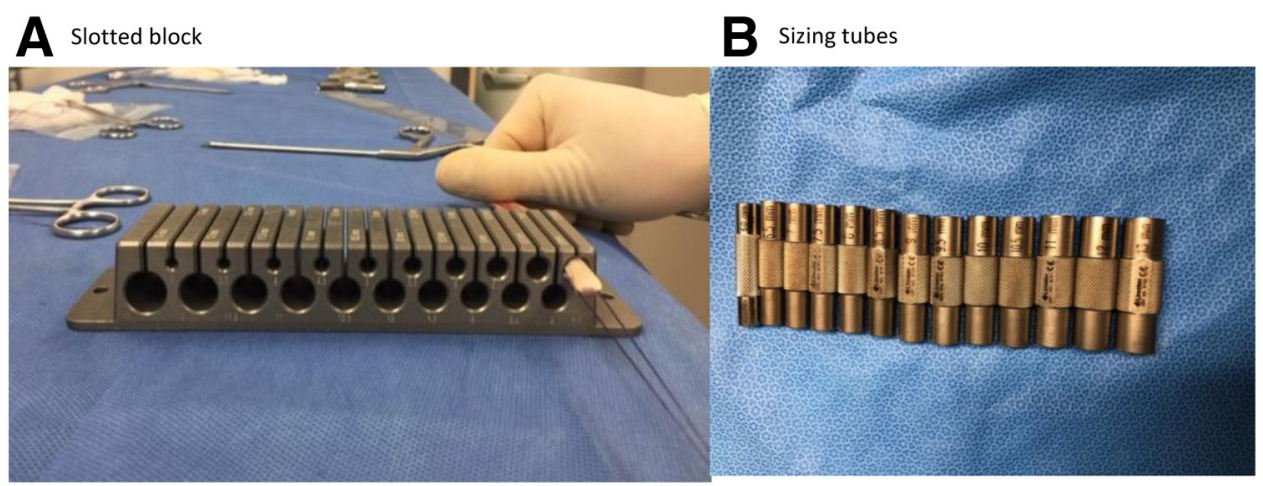

Altman method was used to assess more precisely the agreement between the two devices. This method proposes an alternative analysis, based on the quantification of the agreement between two quantitative measurements by studying the mean difference and constructing limits of agreement. ${ }^{9}$ All statistical analyses were performed using SPSS (v23.0.0 from IBM).

\section{Results}

Ninety-nine graft samples from 33 knees were measured using both sizing tools by two attending surgeons $(n=198)$. The demographic cadaver's information are detailed in Table 1. The intraobserver reliability was excellent with intra-correlation coefficients varying between 0.931 and 0.99 for both the slotted sizing block and the sizing tubes. The interobserver reliability was also good to excellent with a coefficient between 0.897 and 0.953 . Pearson correlation score of $0.991(P<.001)$ was assessed between the 2 measuring tools (see Tables 2 and 3 for detailed results). Moreover, the Bland-Altman graph showed a mean difference of -0.05 , with $95 \%$ limits of agreement between -0.46 and 0.37 . When breaking down per surgeon, surgeon 1 showed a mean difference of 0.01 between the 2 techniques (95\% limits of agreement: $-0.38,0.39)$, whereas surgeon 2 showed a mean difference of -0.10 (95\% limits of agreement: $-0.52,0.32]$ ).

\section{Discussion}

The results of our study showed that slotted sizing block and closed tubes are interchangeable. Adequate ACL graft sizing is extremely important. Accurate graft diameter measurement ensures that the femoral and tibial tunnels will have the optimal size for graft passage

Table 1. Demographic Information

\begin{tabular}{lc}
\hline Number of specimens & $17(33 \mathrm{knees})$ \\
Average age & $76.2 \pm 13.6$ years \\
Body mass index & $22.6 \pm 4 \mathrm{~kg} / \mathrm{m}^{2}$ \\
Sex & $52.9 \%$ males \\
\hline
\end{tabular}

and integration. A study by Spragg et al. ${ }^{5}$ also showed that every $0.5 \mathrm{~mm}$ in graft diameter increment is inversely proportional to graft failure rate. The surgeon could therefore use a threshold diameter value for which to enlarge the graft to lower the failure rate. The harvested graft diameter measurement has therefore to be reliable.

Reliability was first assessed trough intraobserver and interobserver reliability. In this specific case, both the intraobserver and interobserver reliability were excellent, with the 2 surgeons obtaining the same value in more than $80 \%$ of sample measurements. A study by Dwyer et al. ${ }^{10}$ obtained similar results using cadaver hamstrings.

The second aspect of reliability concerns the different measurement tools. Our initial hypothesis was that the slotted sizing block could underestimate the graft diameter because some tendon fibers could potentially escape through the opening. However, in $95 \%$ of cases, the diameter difference between the tubes and the block measures was less than the $0.5 \mathrm{~mm}$ in increment cutoff. Thus if the block does underestimate the graft diameter, the size difference cannot be determined with the resolution of our instruments. This could also explain the fact that the $0.5 \mathrm{~mm}$ increment, which represents one tube size increment, was included in the confidence interval. This could have been avoided with the use of smaller scale instruments $(0.2 \mathrm{~mm}$ increment, for example), which are, to our knowledge, nonexistent.

Our results support therefore that in the context of graft sizing, the open and closed instruments tested can

Table 2. Intrarater and Inter-Rater Reliability

\begin{tabular}{lccllc}
\hline & \multicolumn{2}{c}{ Slotted sizing tool } & & \multicolumn{2}{c}{ Tubes } \\
\cline { 2 - 3 } \cline { 5 - 6 } & ICC & $95 \%$ CI & & ICC & $95 \%$ CI \\
\hline Intrarater reliability & & & & & \\
$\quad$ Surgeon 1 & 0.962 & {$[0.858,0.990]$} & & 0.931 & {$[0.738,0.983]$} \\
$\quad$ Surgeon 2 & 0.972 & {$[0.894,0.993]$} & & 0.988 & {$[0.956,0.997]$} \\
$\quad$ Overall & 0.965 & {$[0.914,0.986]$} & & 0.952 & {$[0.880,0.981]$} \\
Inter-rater reliability & 0.980 & {$[0.970,0.987]$} & & 0.979 & {$[0.950,0.989]$} \\
\hline
\end{tabular}


Table 3. Correlation Slotted Versus Tubes

\begin{tabular}{lccc}
\hline Grafts combination type & $\mathrm{N}$ & Correlation & $P$ \\
\hline $\begin{array}{l}\text { Doubled semitendinosus } \\
\text { and gracilis (4HT) }\end{array}$ & 33 & 0.941 & $<.001^{*}$ \\
$\begin{array}{l}\text { Tripled semitendinosus } \\
\quad \text { and gracilis (6HT) }\end{array}$ & 33 & 0.965 & $<.001^{*}$ \\
$\begin{array}{l}\text { Doubled semitendinosus } \\
\text { and gracilis augmented }\end{array}$ & 33 & 0.961 & $<.001^{*}$ \\
$\quad \begin{array}{l}\text { with unfolded quadriceps } \\
\text { tendon graft (4HT+QT) }\end{array}$ & & & \\
Overall & 99 & 0.978 & $<.001^{*}$ \\
\hline
\end{tabular}

*Statistically significant data.

be considered interchangeable. The strength of this study resides in the number of cadaveric grafts used $(\mathrm{n}=33)$ for a total of 99 graft samples used for measurement.

\section{Limitations}

Comparing measuring devices from only l company (Conmed Medical) is this study's principal limitation. Although we may believe that the engineering of those tools must respect the same standards and precision when it comes to the inner diameter values from one company to the other, the study conclusion cannot be automatically transposed to other ACL ancillaries. Also, there was no predetermined sequence in which the grafts had to be measured. Both observers simultaneously measured the grafts after preparing them. They used the first available sizing tool because they were all used at the same time on a common working table. The lack of an organized, reproducible sizing sequence could lead to precision errors. However, this is the technique used in the operating room setting. The graft diameter is thought to be reduced under continuous compression. ${ }^{11}$ Repetitive measurements may have affected the graft size but the ICC results showed very minimal subclinical impact of creep phenomenon in this study.

\section{Conclusions}

This study showed that the ACL graft diameter measurement does not vary whether a slotted block or sizing tube from the same company is used.

\section{Acknowledgments}

Special thanks to Claudia Beaulieu, Sonia Paquette and Denis Bisson, technicians in the anatomy laboratory, who helped us greatly with the preservation and preparation of samples. We also thank Catherine Raynaud, who helped with data collection, and Sonia Oviedo Chang, who helped with the English translation.

\section{References}

1. Kim S, Bosque J, Meehan JP, Jamali A, Marder R. Increase in outpatient knee arthroscopy in the United States: A comparison of National Surveys of Ambulatory Surgery, 1996 and 2006. J Bone Joint Surg Am 2011;93: 994-1000.

2. Buller LT, Best MJ, Baraga MG, Kaplan LD. Trends in anterior cruciate ligament reconstruction in the United States. Orthop J Sports Med 2015;3:23259671 14563664.

3. Calvo R, Figueroa D, Figueroa F, et al. Five-strand hamstring autograft versus quadruple hamstring autograft with graft diameters 8.0 millimeters or more in anterior cruciate ligament reconstruction: Clinical outcomes with a minimum 2-year follow-up. Arthroscopy 2017;33: 1007-1013.

4. Shelton WR, Fagan BC. Autografts commonly used in anterior cruciate ligament reconstruction. J Am Acad Orthop Surg 201 1;19:259-264.

5. Spragg L, Chen J, Mirzayan R, Love R, Maletis G. The effect of autologous hamstring graft diameter on the likelihood for revision of anterior cruciate ligament reconstruction. Am J Sports Med 2016;44:1475-1481.

6. Snaebjornsson T, Hamrin Senorski E, Ayeni OR, et al. Graft diameter as a predictor for revision anterior cruciate ligament reconstruction and KOOS and EQ-5D values: A cohort study from the Swedish National Knee Ligament Register based on 2240 patients. Am J Sports Med 2017;45:2092-2097.

7. Park SY, Oh H, Park S, Lee JH, Lee SH, Yoon KH. Factors predicting hamstring tendon autograft diameters and resulting failure rates after anterior cruciate ligament reconstruction. Knee Surg Sports Traumatol Arthrosc 2013;21:1111-1118.

8. Figueroa F, Figueroa D, Espregueira-Mendes J. Hamstring autograft size importance in anterior cruciate ligament repair surgery. EFORT Open Rev 2018;3:93-97.

9. Giavarina D. Understanding Bland Altman analysis. Biochem Med 2015;25:141-151.

10. Dwyer T, Whelan DB, Khoshbin A, et al. The sizing of hamstring grafts for anterior cruciate reconstruction: Intra- and inter-observer reliability. Knee Surg Sports Traumatol Arthrosc 2015;23:1197-1200.

11. Cruz A, Fabricant P, Seeley M, Ganley T, Lawrence J. Change in size of hamstring grafts during preparation for ACL reconstruction: Effect of tension and circumferential compression on graft diameter. J Bone Joint Surg Am 2016;98:484-489. 